Published in final edited form as:

Expert Rev Clin Pharmacol. 2018 June ; 11(6): 549-559. doi:10.1080/17512433.2018.1478725.

\title{
Gemtuzumab Ozogamicin for Treatment of Acute Myeloid Leukemia
}

\author{
Jeffrey Baron ${ }^{1}$ Eunice S. Wang ${ }^{2}$ \\ ${ }^{1}$ Department of Pharmacy, Roswell Park Comprehensive Cancer Center, Buffalo, NY, USA \\ 2Leukemia Service, Department of Medicine, Roswell Park Comprehensive Cancer Center, \\ Buffalo, NY, US
}

\begin{abstract}
Introduction: Gemtuzumab ozogamicin (GO) is an antibody-drug conjugate consisting of a monoclonal antibody targeting CD33 linked to a cytotoxic derivative of calicheamicin. Despite the known clinical efficacy in relapsed/refractory acute myeloid leukemia (AML), GO was withdrawn from the market in 2010 due to increased early deaths seen in newly diagnosed AML patients receiving GO + intensive chemotherapy. In 2017, new data on the clinical efficacy and safety of GO administered on a fractionated dosing schedule led to re-approval for newly diagnosed and relapsed/refractory AML.
\end{abstract}

Areas covered: Addition of fractionated GO to chemotherapy significantly improved event-free survival of newly diagnosed AML patients with favorable and intermediate cytogenetic risk disease. GO monotherapy also prolonged survival in newly diagnosed unfit patients and relapsefree survival in relapsed/refractory AML. This new dosing schedule was associated with decreased incidence of hepatotoxicity, veno-occlusive disease (VOD), and early mortality.

Expert commentary: GO represents the first drug-antibody conjugate approved (twice) in the US for AML. Its re-emergence adds a valuable agent back into the armamentarium for AML. The approval of GO as well as three other agents for AML in 2017 highlights the need for rapid cytogenetic and molecular characterization of AML and incorporation into new treatment algorithms.

\section{Keywords}

acute myeloid leukemia; CD33; antibody drug conjugate; fractionated dosing; gemtuzumab ozogamicin

\footnotetext{
Corresponding author: Eunice S. Wang, Roswell Park Comprehensive Cancer Center, Elm and Carlton Streets, Buffalo, NY 14263; Eunice.wang@ roswellpark.org; Tel: (716) 845-3544; Fax: (716) 845-4620.

Declaration of Interest

J Baron and ES Wang are advisory board members for Pfizer. The authors have no other relevant affiliations or financial involvement with any organization or entity with a financial interest in or financial conflict with the subject matter or materials discussed in the manuscript apart from those disclosed. Peer reviewers on this manuscript have no relevant financial or other relationships to disclose. Pfizer provided a scientific accuracy review at the request of the journal.
} 


\section{Overview of the market}

Acute myeloid leukemia (AML) is the most common form of aggressive leukemia, accounting for $80 \%$ of acute leukemias in adults with a median age at diagnosis of 67 years in the US population.(1) AML is a hematologic malignancy characterized by rapid clonal expansion of undifferentiated myeloblasts in the peripheral blood and/or bone marrow. For fit individuals, standard upfront therapy remains cytarabine and anthracycline based intensive chemotherapy (7+3).(2-9) For those unable to receive intensive chemotherapy, therapy with hypomethylating agents (HMA), azacitidine or decitabine, is indicated with lower remission rates but decreased therapy-related mortality. Despite these interventions, the majority of patients will succumb to disease relapse or therapy-related mortality, leading to reported 5-year overall survival rate among all AML patients in the US of approximately $26 \%$.(10) Outcomes are impacted by the overall comorbidities and functional status of patients as well as by the presence of myriad driver and secondary mutations and cytogenetics predicting chemotherapy responses.(10)

2017 ushered in a new therapeutic era for AML with the much-anticipated approval of four treatment modalities for this disease. Enasidenib was approved for the treatment of relapsed/ refractory AML (RR-AML) characterized by $I D H 2$ mutations (estimated $\leq 10 \%$ of patients). Midostaurin was approved in combination with upfront chemotherapy for newly diagnosed AML (ND-AML) characterized by FLT3 mutations (approximately one third of patients). Liposomal cytarabine and daunorubicin formulation was approved for patients with secondary (s-AML), therapy-related ( $\mathrm{t}-\mathrm{AML})$, and AML with myelodysplastic related characteristics (AML-MRC) (20-25\% of patients).

Gemtuzumab ozogamicin (GO) is the only new agent approved for treatment of the vast majority of AML patients, including ND-AML and RR-AML disease in adults and RRAML in pediatric patients. Of note, these indications for GO expand upon the previous US approval of GO, which was limited only to adult patients with RR-AML in 2000. The reemergence of this agent in 2017 represents a therapeutic breakthrough and validates the efficacy and safety of a novel fractionated and lower dose treatment regimen. We review here the clinical pharmacology, mechanism of action, clinical trial results, and rationale for the re-approval of this agent.

\section{Introduction to the Drug}

\subsection{Clinical Pharmacology}

GO is an antibody-drug conjugate (ADC) consisting of an antibody directed against human CD33 (gemtuzumab) linked to a cytotoxic component (ozogamicin). The transmembrane surface receptor, CD33, is an attractive target for AML as it is nearly ubiquitously expressed on hematopoietic cells of myeloid lineage and myeloblasts in $>80 \%$ of AML patients.(11) This agent was designed theoretically to enhance directed anti-leukemic efficacy with less non-specific toxicity against normal tissues. Gemtuzumab is a recombinant humanized IgG4 kappa antibody with high affinity to the CD33 receptor without intrinsic anti-leukemic activity. This antibody is attached via a specific linker to the cytotoxic derivative of calicheamicin. Once bound to CD33, the ADC is internalized. Following internalization, GO 
is trafficked from endosomes to lysosomes where linker cleavage occurs in the acidic environment of the lysosome. The free calicheamicin derivative now trafficks to the nucleus, where it binds to DNA and induces double strand breaks, leading to cell cycle arrest and apoptosis.(12) Theoretically pluripotent stem cells and non-hematopoietic cells are not affected by GO as they lack CD33 expression.

GO was initially studied as a $9 \mathrm{mg} / \mathrm{m}^{2}$ dose administered as a 2-hour intravenous infusion every 14 days for 2 doses per month. Following injection, GO displays non-linear drug pharmacokinetics with a volume of distribution of approximately $21.4 \mathrm{~L}$ in patients. In vitro studies showed that calicheamicin itself is extensively bound to human plasma proteins at $97 \%$ and metabolized through non-enzymatic reduction of the disulfide moiety which then undergoes elimination. The terminal half-life of GO is 62 hours after the first $9 \mathrm{mg} / \mathrm{m}^{2}$ dose and 90 hours after the second $9 \mathrm{mg} / \mathrm{m}^{2}$ dose.

Following its withdrawal from the commercial market in 2010 (discussed below), GO continued to be studied as a single agent and in combination with chemotherapy at different doses as well as in a fractionated schedule. This latter regimen delivered less total drug with corresponding less toxicity but was hypothesized to retain clinical efficacy based on the biology of CD33 receptor kinetics. After internalization, CD33 receptors are recycled and re-expressed on surface of the myeloblasts in approximately 72 hours. Use of a fractionated schedule every 3 days therefore could provide $>90 \%$ saturation of re-expressed CD33 receptors and theoretically would result in more consistent anti-leukemic activity as compared with 14-day dosing despite lower total doses of administered drug. $(13,14)$ Although clinical pharmacokinetic data is not known for the fractionated regimen, per se, one can extrapolate drug kinetics based on the original dosing regimen. Following a 9 $\mathrm{mg} / \mathrm{m}^{2}$ dose, high $\mathrm{C}_{\max }$ levels may be responsible for the increased liver toxicity seen with the original GO dosing schedule. This could theoretically be mitigated by using a fractionated dosing schedule of $3 \mathrm{mg} / \mathrm{m}^{2}$ per dose, which effectively reduces $\mathrm{C}_{\max }$ levels by up to $75 \%$. $(15,16)$

\section{Clinical Efficacy}

\subsection{Relapsed/Refractory AML (Non-fractionated Dosing)}

The clinical efficacy of GO was first established in 3 open label, single-arm Phase II studies of AML in first relapse.(17) All three studies were of similar design with slightly different inclusion criteria. In general, adult patients $\geq 18$ years old with AML in first relapse received monotherapy with two doses of $\mathrm{GO}$ at $9 \mathrm{mg} / \mathrm{m}^{2} /$ dose administered as a 2-hour intravenous infusion separated by 2 weeks. Patients were evaluated for response, survival, and treatmentemergent adverse events. In total, 277 patients (median age 61 years old) received GO treatment. Overall response rate (ORR) consisting of complete remission (CR) and complete remission with incomplete hematologic recovery (CRi) was 26\% (71 patients): CR in 35 patients (13\%), and CRi in 36 patients (13\%). Median relapse-free survival (RFS) was 5.2 months. Median overall survival (OS) was 12.6 months for those that responded to GO. Based on this evidence of single-agent efficacy, GO received accelerated approval in 2000 by the United States Federal Drug Administration (FDA) using this $9 \mathrm{mg} / \mathrm{m}^{2}$ dosing 
regimen; however the FDA required additional post-marketing studies, including a randomized trial, to further confirm efficacy.(18)

An open-label dose escalation study also established the efficacy of GO in pediatric patients with RR-AML.(19) Twenty-nine children ranging in age from 1-16 years received GO in doses ranging from $6-9 \mathrm{mg} / \mathrm{m}^{2} /$ dose for 2 doses separated by 2 weeks. Additional inclusion criteria included CD33 receptor presence on blast cells by immunofluorescence staining. Of note, patients who had undergone previous allogeneic stem cell transplant (alloSCT) were excluded. Eight out of 29 patients (28\%) achieved a CR with similar responses in those patients with primary refractory (30\%) vs. relapsed disease (26\%). Thirteen patients $(45 \%)$ of this original cohort underwent subsequent alloSCT after GO. The efficacy of GO was confirmed in an additional 96 patients whose ages ranged from 0.2 to 21 years old. Based on these studies, GO was FDA approved for pediatric RR-AML in individuals aged $\geq 2$ years old.(14)

\subsection{Newly diagnosed AML (Non-fractionated dosing)}

In accordance with this FDA accelerated-approval process, the SWOG national cooperative group initiated a phase 3 randomized controlled trial (SWOG-S0106) of GO at a dose of 6 $\mathrm{mg} / \mathrm{m}^{2}$ combined with induction chemotherapy vs. chemotherapy alone in ND-AML adult patients. Of note, each arm of this trial utilized a different daunorubicin dose. Patients randomized to the GO arm received daunorubicin (D) dosed at $45 \mathrm{mg} / \mathrm{m}^{2}$ for 3 days combined with infusional cytarabine (A) and GO dosed at $6 \mathrm{mg} / \mathrm{m}^{2}$ on day 4 . Patients in the control arm (DA) received a higher daunorubicin dose of $60 \mathrm{mg} / \mathrm{m}^{2}$ for 3 days combined with cytarabine. GO was also added to day 1 of high dose cytarabine consolidation. This study was stopped prematurely due to interim data demonstrating a lack of clinical benefit and increased toxicity associated with the GO+DA arm. The results failed to show any improvement in complete response rate, RFS, or OS rates when GO was added. Moreover, a significantly higher mortality rate of $5.5 \%$ was reported in the GO+DA vs. $1.4 \%$ in the DA arm $(p=0.01)$. (20) This increased mortality occurred during induction chemotherapy and was not related to increased liver function tests or sinusoidal obstructive syndrome. Six of the 16 deaths in the GO+DA arm resulted from hemorrhage (4 central nervous system, 2 pulmonary). Five patients died of infection and/or febrile neutropenia. Based on these results, GO was voluntarily withdrawn from the US commercial market in June 2010.(21) It is important to note that this phase 3 trial utilized a completely different trial design and patient population than the original Phase 2 trials and was the first trial to study GO combined with chemotherapy as upfront therapy. The use of what is now known to be an inferior daunorubicin dose $\left(45 \mathrm{mg} / \mathrm{m}^{2}\right)$ in the GO+DA arm is now thought to have negatively influenced outcome in this group independent of GO. Moreover the mortality rate of $1.4 \%$ in the DA arm was much lower than was reported in similar phase 3 AML trials involving the same induction regimen.

\subsection{Relapsed/refractory AML (Fractionated Dosing)}

MyloFrance-1 was an investigator-initiated phase 2 single-arm open-label trial evaluating the use of a fractionated dosing schedule of GO in CD33+ AML patients in first relapse with a CR duraction of 3-18 months.(22) Any expression of CD33 on myeloblasts was allowed. 
Patients with s-AML, AML-MRC, APL, or prior stem cell transplant (SCT) were excluded. Treatment consisted of a GO monotherapy given as $3 \mathrm{mg} / \mathrm{m}^{2}$ on Days 1,4 , and 7. Patients achieving a response proceeded onto consolidation therapy without further GO. Although SCT following GO was permitted, a delay of at least 90 days between GO and transplant was required. Among 57 patients (median age 64 years (range 22-80 years)), 15 patients (26\%) achieved CR and 4 (7\%) achieved CRi following a single course of GO, leading to a ORR (CR/CRi) of $33 \%$. No significant differences were noted based on age $\geq 60$ years old, cytogenetic risk groups, and duration of first remission $\leq 12$ months. Median RFS was 11.0 months, and median OS was 8.4 months. Overall GO was well tolerated without any evidence of grade 3 or 4 hepatotoxicity and median duration of cytopenias of 21-23 days. Seven patients received subsequent transplant (3 allogeneic, 4 autologous) after GO treatment with a median time between GO and transplant of 5 months (range 112-220 days). No veno-occlusive disease (VOD) (0\%) was observed in any patients including those who underwent alloSCT.

\subsection{Newly Diagnosed AML (Fractionated Dosing)}

Combination GO and chemotherapy: Based on the favorable efficacy and toxicity profile, this fractionated dosing regimen was then studied in combination with upfront induction and consolidation chemotherapy for ND-AML in fit older adults in the ALFA-0701 study, a multi-center, randomized open-label phase 3 trial of patients aged 5070 years old.(14) Patients were randomized in a 1:1 fashion to receive induction therapy consisting of GO dosed at $3 \mathrm{mg} / \mathrm{m}^{2}$ (up to maximum of one vial) on days 1,4 , and 7 plus induction chemotherapy $(n=135)$ vs. chemotherapy alone $(n=136)$. Induction consisted of daunorubicin $\left(60 \mathrm{mg} / \mathrm{m}^{2}\right.$ on Days $\left.1-3\right)$ and cytarabine (200 $\mathrm{mg} / \mathrm{m}^{2}$ on Days $\left.1-7\right)$ (DA) in both groups. Patients with refractory disease could receive a second DA induction alone. Patients achieving $\mathrm{CR} / \mathrm{CRi}$ received consolidation therapy according to their initial randomization with 2 courses of GO dosed at $3 \mathrm{mg} / \mathrm{m}^{2}$ (up to a maximum of one vial) on day 1 plus daunorubicin $\left(60 \mathrm{mg} / \mathrm{m}^{2}\right.$ on day 1 of course $1 ; 60 \mathrm{mg} / \mathrm{m}^{2}$ on days 1 and 2 of course 2$)$ and cytarabine $\left(1 \mathrm{~g} / \mathrm{m}^{2}\right.$ every 12 hours on days $\left.1-4\right)$ or chemotherapy alone. The primary endpoint was event-free survival (EFS). Secondary endpoints were ORR, OS, and RFS. Patients eligible for HSCT had a recommended interval of at least 2 months between the last dose of $\mathrm{GO}$ and transplantation. Results of 278 patients enrolled on this trial were initially published in 2012 by Castaigne and colleagues.(23) This data recently updated to include 271 patients for analysis and excluding 9 patients due to lack of informed consent. (24) Overall, the ALFA-0701 study confirmed the clinical benefit of GO in the majority of ND-AML patients. In the original publication, median age was 62 years. Baseline cytogenetics demonstrated that 3\% (9/278) had favorable risk, 65\% (182/278) had intermediate risk, $21 \%$ (58/278) had poor/adverse risk, and 10\% (29/278) could not be classified. The addition of GO to DA did not significantly improve response rates $(\mathrm{OR}=$ 1.46, 95\% CI 0.82-2.59; $\mathrm{p}=0.25$ ) despite less refractory disease and increased CRi in the GO arm. CR/CRi rates were $81 \%$ in GO+DA vs. $75 \%$ in the DA arm. However, median EFS was significantly higher (15.6 months) in the GO+DA arm vs. DA control (9.7 months) with a hazard ratio (HR) of 0.58 (95\% CI: $0.43-0.73$; p value $=0.0003$ ). Two-year EFS was $40.8 \%$ in the GO+DA group vs. $17.1 \%$ with DA only. Forty patients went on to receive alloSCT in first CR/CRi whereas 25 patients received alloSCT without CR/CRi or following relapse; 
differences in terms of EFS remained significant after accounting for alloSCT. (23)

Subgroup analyses confirmed that improvement in EFS was observed in all patients receiving GO when stratified by age, favorable/intermediate risk cytogenetics, FLT3-ITD and NPM-1 mutant status. Of note, only AML patients with poor risk cytogenetics did not seem to benefit from GO. Subsequent post-hoc analyses suggested the clinical benefit of GO may have been particularly pronounced in patients with normal karyotype FLT3-ITD mutant AML as well as NPM-1 mutant disease as evidenced by lower rates of detectable minimal residual disease in molecular assays.(25, 26) Importantly, induction mortality (i.e. within 28 days after the last dose of study treatment) did not differ between each arm: $9(6 \%)$ following $\mathrm{GO}+\mathrm{DA}$ vs. $5(4 \%)$ in the DA arm $(\mathrm{p}=0.41)$. Hematologic toxicity, in particular persistent thrombocytopenia, was more common following GO than control [22 (16\%) vs. 4 $(3 \%) ; \mathrm{p}<0.0001]$. There was a higher but not statistically significant incidence of grade 3-4 hemorrhage as a result of the persistent thrombocytopenia. Other toxicities, including cardiac events, infectious events, transfers to intensive care unit, deaths from toxic events, and grade 3-4 hepatotoxicity due to sepsis did not differ significantly between the two arms. VOD occurred only in 3 patients who received GO for an incidence of $2 \%$. Overall Castaigne and colleagues concluded that use of fractionated GO in combination with standard intensive chemotherapy improved EFS and OS in ND-AML patients and was well tolerated with no new safety concerns.(23)

As noted above, an updated analysis of the ALFA-0701 trial including 271 patients was recently presented by the pharmaceutical sponsor as part of the recent USA approval of this drug. $(15,24)$ In this update, the ORR $(\mathrm{CR}+\mathrm{CRp})$ by investigator assessment was not significantly different between patients in the GO+DA arm (81.5\%) vs the DA arm (73.5\%) (risk difference of 7.95 [95\% CI: $-3.79-19.85]$ with $p=0.1457$ using Fisher's exact test). As a result, there is no mention of CR/CRp in the USA drug label. Although the original report of the ALFA-0701 trial reported a statistically significant improvement in OS (with a median OS of 34 months after GO +DA vs. 19.2 months in the control arm), the updated FDA document reported no significant difference in OS between the two groups presumably due to longer follow-up time. However a remarkable difference in EFS remained with a median EFS of 17.3 months vs 9.5 months in the GO vs non-GO arms (HR 0.56, 0.42-0.76, p<. 001). Three year EFS was $39.8 \%$ vs $13.6 \%$ (HR 0.56, 0.42-0.76, p<.001). Subgroup analyses stratified for EFS and OS by age, sex, ECOG, CD33 expression, and cytogenetic risk categories demonstrated benefit for all patients except those with AML with adverse cytogenetics. $(15,16)$

Further confirmation of the clinical efficacy of GO in the upfront setting with chemotherapy was demonstrated in a meta-analysis summarizing the results of over 3300 patients enrolled on 5 randomized controlled trials for previously untreated AML.(27) Primary endpoint for the meta-analysis was OS with secondary endpoints of ORR, relapse risk, RFS, and safety. All data was analyzed using standard meta-analytic techniques with a fixed-effect method. As detailed in the original publication by Hills and colleagues, a total of 3325 patients across five randomized controlled trials up to May 1, 2013 were included (Tables 1-2). Ages ranged from 15-84 years old (median 58 years), and 55\% (1842 of 3325) were male. Eightyeight percent (2927 of 3325) had ND-AML whereas 9\% (285 of 3325) had s-AML and 3\% (113/3325) had high-risk myelodysplastic syndrome (MDS). GO was administered at 3-6 
$\mathrm{mg} / \mathrm{m}^{2}$ per dose on different schedules: $3 \mathrm{mg} / \mathrm{m}^{2}$ on day 1 in two trials, $6 \mathrm{mg} / \mathrm{m}^{2}$ on day 4 in 2 trials, and $3 \mathrm{mg} / \mathrm{m}^{2}$ (maximum dose $5 \mathrm{mg}$ ) on days 1, 4, and 7 (ALFA-0701). No trial utilized the $9 \mathrm{mg} / \mathrm{m}^{2}$ dose. (27)

Overall, this meta-analysis confirmed the clinical efficacy of GO. In the original Hills publication, although there was no detectable difference in ORR (CR/CRi) between GO vs non-GO containing arms, the addition of GO was associated with significantly decreased relapse risk and improved 5 year OS. Absolute benefit was most apparent in patients with AML characterized by favorable and intermediate (but not adverse) risk cytogenetics. In an updated analysis presented in FDA documents including 3331 patients, a clear improvement in median OS was noted (GO 23.6 vs. no GO 21.5 months, OR 0.90, CI 0.82-0.98; p=0.01). GO was also associated with significant improvement in EFS (9.6 vs. 7.6 months, OR 0.85, 95\% CI 0.77-0.94; $\mathrm{p}=0.0002)$ and RFS (18.1 vs. 14.5 months, OR 0.8, 95\% CI 0.76-0.92; $\mathrm{p}=0.0003)$. $(15,16)$ Further sub-group analysis again revealed that patients with AML characterized by favorable cytogenetics most benefited from the addition of GO with less but still significant survival benefit in AML with intermediate cytogenetics. No survival benefit was seen in patients with AML with adverse/poor-risk cytogenetics. Notably, the increased early induction mortality reported in the SWOG S0106 study with GO dosed at $6 \mathrm{mg} / \mathrm{m}^{2}$ on day 4 was not replicated in the other trials of GO utilizing t $3-6 \mathrm{mg} / \mathrm{m}^{2}$ with chemotherapy. However, the finding that GO dosed $6 \mathrm{mg} / \mathrm{m}^{2}$ was associated with increased 30 day mortality as compared with the $3 \mathrm{mg} / \mathrm{m}^{2}$ dose supports that use of lower doses of GO as a safer modality to retain clinical efficacy while mitigating the risk of life-threatening toxicities in combination with chemotherapy. Evidence of decreased relapse risk associated with the fractionated dosing over the non-fractionated dosing regimens was also reported. This meta-analysis also highlighted the fact that the SWOG S0106 trial was in many ways an outlier. All of the other 4 trials administered GO on day 1 instead of day 4 and utilized full dose daunorubicin dosing with overall mortality rates higher than $1.4 \%$ in the control arms. Three trials administered GO during both induction and consolidation. Most importantly all of the trials except the S0106 study demonstrated a benefit to adding GO to standard chemotherapy without increased toxicity or early mortality (Tables 1 and 2).

Monotherapy in unfit newly diagnosed AML: Amadori and colleagues conducted the randomized AML-19 study to investigate the efficacy of fractionated GO as monotherapy in ND-AML patients unfit for aggressive chemotherapy.(28) This multi-center randomized open-label phase 3 study compared GO to best supportive care (BSC) for patients with NDAML. Individuals were either $>75$ years old or 61-75 years old with a World Health Organization performance status (WHO PS) $>2$ or were unwilling to receive intensive chemotherapy. Patients were randomized in a 1:1 fashion to GO vs. BSC. GO was dosed at 6 $\mathrm{mg} / \mathrm{m}^{2}$ on day 1 followed by a second dose at $3 \mathrm{mg} / \mathrm{m}^{2}$ on day 8 during induction. Patients with no evidence of disease progression or significant toxicities after induction were eligible to receive continuation therapy with a single monthly dose of GO $2 \mathrm{mg} / \mathrm{m}^{2}$ every 4 weeks for up to 8 courses. BSC included standard supportive care measures, hydroxyurea, or other anti-metabolites. Because of the timing of this trial, HMA (azacitidine, decitabine) were not included in the BSC arm. In total, 237 patients were randomized: 118 to treatment with GO, and 119 to BSC. Most patients were very elderly with a median age of 77 years (range, 62- 
88) and a baseline WHO PS of $0-1$ (65\% of patients). Compared to BSC, patients receiving GO arm were more likely to be women (52\% vs. $39 \%$ ) with favorable/intermediate risk cytogenetics (50\% vs. $38 \%$ ). GO significantly improved OS (HR 0.69; 95\% CI: $0.53-0.90$; $\mathrm{p}=0.005$ ) with a median OS of 4.9 months vs. 3.6 months with BSC. The OS benefit was most apparent in those with high CD33 blast expression, favorable/intermediate cytogenetics, and in women. Overall responses ( CR/CRi) occurred in 27\% (30 out of 111) patients receiving GO. No new safety concerns were noted with use of GO with similar rates of serious adverse events between the two groups.

\section{Safety and Toxicity}

The current indication for GO contains a black box warning for hepatotoxicity including potential fatal or severe VOD (also known as sinusoidal obstructive syndrome (SOS)). Although VOD/SOS was noted in subsequent or prior recipients of HSCT following the original $9 \mathrm{mg} / \mathrm{m}^{2}$ dosing, the incidence of this complication has been markedly reduced with lower dose fractionated dosing. In the ALFA-0701 trial, although $>50 \%$ of patients developed hyperbilirubinemia of any grade following GO+DA, VOD occurred in only 5\% (6 of 131) patients. Median time to onset of VOD from GO dosing was 9 days. Five patients developed VOD within 28 days of GO dosing; three of the 6 cases were fatal. Additionally2 cases of VOD occurred in patients in the control group who subsequently received GO for relapsed disease. No patients with RR-AML treated with single agent GO in the MyloFrance-1 trial developed VOD even after undergoing HSCT. Of note, an interval of 2-3 months was recommended between last GO dose and HSCT in both the ALFA-0701 and MyloFrance trials. In summary, analysis of GO across all AML trials confirms a significantly higher incidence of VOD correlating with higher GO dose, and baseline hepatic impairment, and prior or subsequent HSCT. Contemporary regimens utilizing GO doses of $3-6 \mathrm{mg} / \mathrm{m} 2$ are consistently associated with a VOD/SOS incidence of $0-5 \%$.

Other notable toxicities of GO include myelosuppression which was almost universal as well as infusion related reactions occurring during or within 24 hours of therapy. Persistent thrombocytopenia has been associated with increased rates of hemorrhage. Management consists of frequent monitoring and holding drug for prolonged neutropenia and/or thrombocytopenia. Premedication is required prior to all GO doses with discontinuation of drug for potentially life-threatening anaphylactic reactions.

\section{Biomarkers of Response}

Single nucleotide polymorphisms (SNPs) for the CD33 receptor have been proposed as potential biomarkers of response to GO. The rs $12459419 \mathrm{C}>\mathrm{T}$ polymorphism in the splice enhancer region regulates an alternatively spliced CD33 receptor expressing the CD33IgV domain (the antibody-binding site for GO) due to a lack of exon2 (D2-CD33). As a result, GO cannot bind to CD33. Lamba and colleagues evaluated the impact of this polymorphism on clinical response to GO + chemotherapy vs. chemotherapy alone response in de novo pediatric AML patients as part of the Children's Oncology Group AAML0531 trial.(29) Three genotypes were identified: CC genotype (wildtype) in 415 patients (51\%), CT genotype (SNP) in 316 patients (39\%) and TT genotype (SNP) in 85 patients (10\%). Patients 
with the CC genotype had significantly lower relapse risk in the GO arm vs. chemotherapy alone (26\% vs. $49 \%$; p-value $=0.001)$ and improved disease-free survival (GO 65\% vs. $46 \%$; p-value $=0.004$ ). This benefit of GO was not seen in patients with CT or TT genotype, suggesting that the CC genotype for rs12459419 represents a potential means of selecting patients for GO therapy. However a recent re-analysis of the results of the UK AML trials for CD33 splicing genotypes revealed no correlation between these SNPs and responses. (30) Given these conflicting results, testing of SNPs for CD33 receptor outside of clinical trials is not routinely performed at most centers due to cost and uncertainty regarding its clinical benefit.

The activity of GO also likely depends upon CD33 expression by AML blasts. Pollard and colleagues performed an exploratory sub-group analysis of the AAML0531 trial evaluating clinical response stratified by level of CD33 expression (by multi-dimensional flow cytometry per 4 quartiles based on mean fluorescent intensity (MFI)). Those with low CD33 expression (Q1, median MFI 34.61, range 2.68-67.00) had no clinical benefit with GO plus chemotherapy (relapse risk: GO 36\% vs. No GO 34\%, p-value $=0.731$; EFS: GO 53\% vs. No GO 58\%, p-value $=0.456)$. However those with higher CD33 expression (Q2-4) had significantly reduced relapse risk (GO $32 \%$ vs. No-GO 49\%; p-value <0.001) and improved EFS (GO 53\% vs. 41\%; p-value=0.005). (31) Olombel and colleagues performed similar retrospective analyses on patients enrolled in the ALFA-0701 trial. They not only confirmed prior studies that high CD33 expression was associated with higher white blood cell count, marrow blast disease, FLT3-ITD and NPM1 mutations, but also stated that a CD33 ${ }^{+}$ expression level of $\geq 70 \%$ was necessary for a beneficial response to GO, independent of mutational status.(32) Unfortunately, CD33 measurement by flow cytometry is not standardized, and the clinical trials with GO have utilized different requirements for baseline CD33 expressions for eligibility and retrospective analyses. Additional prospective trials are needed before clinicians can utilize the degree of CD33 expression levels for treatment decisions.

Given that GO consists of an antibody linked to cytotoxic chemotherapy, it is not surprising that more conventional mechanisms of chemotherapy resistance can affect response. Multidrug resistance (MDR) mediated by ATP-dependent drug transporters such as Pglycoprotein and MDR-related proteins also correlate with preclinical responses to GO therapy. This was shown clinically in a trial of 29 pediatric RR-AML patients where the relative degree of drug efflux mediated by ex vivo patient samples predicted for response. Five of 8 patients with low drug efflux ratios achieved CR/CRp as opposed to none of the patients with high drug efflux levels.(19) Other studies have suggested that interpatient variability to calicheamicin may also influence GO response.(33)

\section{Conclusion}

Despite the negative results and stigmatism associated with GO, its mechanism of action of this ADC provide a unique means of targeting AML cells with theoretically less toxicity than conventional chemotherapy. Re-approval of this agent for AML patients based on the development of a novel fractionated dosing schedule which preserves the clinical benefit of GO while mitigating the incidence of VOD/SOS (from 20-40\% to now 0-5\%) (see Tables). 
$(13,14) .(15,22,23,28)$ As of September 2017, GO administered at $3-6 \mathrm{mg} / \mathrm{m}^{2}$ per dose is now standard of care therapy for the following indications: 1) as monotherapy in CD33+ RR-AML patients aged 2 years and older; 2) in combination with standard cytarabine and daunorubicin induction and consolidation in de-novo (not secondary or AML-MRC) CD33+ AML patients; 3) as single therapy in CD33+ adult ND-AML patients unfit for intensive chemotherapy.(15)

\section{Expert Commentary}

Although GO $( \pm \mathrm{DA})$ is now considered mainstay treatment for AML, widespread use of this agent for the majority of AML patients must take into consideration some important issues. In ND-AML patients, timely cytogenetic and molecular profiling prior to day 1 of induction therapy is crucial. In the meta-analysis and in other studies, subgroup analysis suggested that GO did not benefit patients with AML characterized by unfavorable cytogenetics. (27) The decision to use GO needs to be made prior to initiating $7+3$ as the approved dosing requires that drug be administered concomitantly with induction chemotherapy on day 1 . In addition, the decision to use liposomal cytarabine and daunorubicin and/or to add midostaurin immediately following 7+3 also depends upon identification of AML with MDS related cytogenetics or FLT3 mutation, respectively. Although in the past, an AML diagnosis was considered a medical emergency prompting urgent initiation of chemotherapy, in the present era, it may be more prudent to wait. Bertoli and colleagues conducted a retrospective cohort of 599 patients with AML from 2000 2009 and concluded that prolonged time from diagnosis to treatment (TDT) (initiation of intensive chemotherapy) did not adversely affect outcomes. The median TDT of 8 days (range, $4-16)$, had no impact on overall survival $(\mathrm{p}=0.4095)$ by multivariate analysis compared with age, s-AML, performance status and ELN risk group and was not negatively associated with response rates and early death.(34) These data provide strong rationale supporting a short waiting period to obtain cytogenetics and molecular information prior to initiation of intensive chemotherapy. $(23,27)$ At many centers, fluorescent in situ hybridization (FISH) for the most common karyotypic abnormalities (i.e., core binding factor lesion) is often the most efficient approach to obtain cytogenetic information for AML diagnoses. In the absence of karyotypic information, it must be noted that the $60-70 \%$ of ND patients under the age of 60 are expected to have favorable or intermediate risk cytogenetics, and the outcomes of GO + DA were not significantly worse than 7+3 alone in adverse cytogenetic AML; therefore initiation of GO prior to the karyotypic information may still be appropriate in majority of younger AML patients.

It remains to be seen where GO will fit overall in the treatment algorithm for ND-AML patients unfit for intensive chemotherapy, The approval of GO monotherapy for unfit ND elderly AML patients was based on a clinical trial demonstrating a survival benefit compared to best supportive care, the latter of which did not include HMA or low-dose cytarabine (LDAC). At present, there are no studies directly comparing GO to HMA or LDAC. When evaluating individual studies, GO and HMA result in similar slightly higher CR/CRi rates of 20-30\% than LDAC (18\%); however some studies have shown longer duration of overall survival (7-9 months) following HMA than GO (5.6 months). (35-37) Logistically, GO has the distinct advantage of being administered as a single IV infusion 
every 2-4 weeks as opposed to the 5-10 daily per month administration of HMA therapy. Although HMA induce less severe and prolonged cytopenias, this direct cytotoxicity of GO results in rapid, more effective cytoreduction and disease control in hyperproliferative AML than HMA. Mechanistically, the mechanism of action of HMA in altering epigenetic processes associated with AML pathogenesis make these agents attractive in treatment of specific molecular subtypes. Decitabine therapy has been shown to result in high responses in AML characterized by complex cytogenetics/TP53 mutations. $(37,38)$ Recently the combination of the potent bcl-2 inhibitor, venetoclax with LDAC or HMA, has resulted in impressively high response rates of 60-70\% in most unfit older AML patients with lower responses (40-50\%) in unfavorable cytogenetics. For defined molecular subsets of disease, targeted FLT3 and IDH1/2 inhibitors offer the convenience of an oral agent with prolonged survival and are now being investigated in combination with both intensive and low dose (HMA, LDAC) chemotherapy. Lastly, the choice of therapeutic approach (GO, HMA, LDAC, venetoclax, IDH1/2 inhibitors) should ideally take into account the patient's wishes regarding aggressiveness, frequency of treatment, and quality of life.

In the RR-AML setting, it is uncertain how often GO monotherapy will be used as compared with standard intensive salvage options (i.e. CLAG-M, MEC,) or non-intensive options (HMA or LDAC). The advantages of GO mirror those for the unfit ND-AML setting, namely infrequent dosing, ease of administration, and more rapid cytoreduction than HMA therapy. The same challenges remain, specifically lower response rates, less targeted effects, and increased toxicities, especially when compared with targeted inhibitors. More than likely, GO will find a place in combination regimens. To date, the results of combination HMA + GO therapy have slightly improved response rates but not overall survival durations. $(39,40)$ Results of GO plus multi-agent intensive chemotherapy (other than daunorubicin and cytarabine) have resulted in impressive response rates and holds promise, particularly in reducing disease burden degree and allowing for subsequent successful alloSCT in the salvage setting.(41-43) Larger prospective confirmatory studies of GO added to targeted therapies agents are needed to more firmly establish GO in the treatment paradigm for the RR-AML population.

\section{Five-year View}

The (re) introduction of GO into the armamentarium for AML has changed the treatment landscape. With the availability of four new drugs including GO, AML treatment now has truly moved into the era of targeted therapies. While it is difficult to accurately predict the status of AML treatment over the next five years, we propose the following scenarios based on the current literature. First, all AML diagnoses will include standard comprehensive molecular, cytogenetic, and flow cytometric analyses in the upfront setting with results available within 24-72 hours. The choices for intensive vs. non-intensive therapy will be based on algorithms for functional status and in-depth patient discussion. The presence or absence of driver mutations (i.e. FLT3, IDH, TP53) as well as expression of surface antigens (CD33, CD123) and cytogenetic risk category will determine the selection of targeted therapy. For ND-AML suitable for intensive chemotherapy, use of GO will be limited to favorable and intermediate cytogenetic risk stratifications. $(23,27)$ Favorable cytogenetic risk AML will be treated with a combination of $7+3$ plus GO and potentially c-kit inhibitors 
which have combined with $7+3$ with improved outcomes. (44). Normal karyotype AML will be treated with $7+3$ in addition to $I D H 1 / 2$ or FLT3 inhibitors plus GO. Adverse karyotype AML will be treated with HMA plus inhibitors for $I D H 1 / 2$, spliceosomes, or FLT3 based on the molecular profiling without additional GO. Unfit older individuals suitable for less intensive therapy will receive a single dose of GO for control of hyperproliferative disease with high white blood cell count at presentation. This will be followed by venetoclax and HMA (in adverse cytogenetics) or LDAC (favorable/intermediate cytogenetics) in patients without specific molecular aberrations (Figure 1). In the RR-AML setting, GO will routinely be added to intensive salvage re-induction chemotherapy regimens (i.e. FLAG, CLAG, MEC) or HMA therapy for fit individuals or utilized as monotherapy in addition to targeted inhibitor therapy for less fit individuals (Figure 2). Overall, based on the improved toxicity profile and significant survival advantage, we anticipate that fractionated GO will become a mainstay treatment option for treatment of AML over the next five years.

\section{Acknowledgments}

Funding

The work of the authors was supported by Roswell Park Comprehensive Cancer Center and National Cancer Institute (NCI) grant P30CA016056. ESW is also supported by the Roswell Park Alliance Foundation (Jacquie Hirsch Leukemia Research Fund).

\section{References}

Clinical trials mentioned in the above review as validating the clinical efficacy and decreased hepatotoxicity of fractionated GO therapy for diverse populations of AML patients are highlighted in the below references by ** (two asterisks). Other articles of interest to the clinical development of GO are indicated by * (one asterisk).

1. Siegel RL, Miller KD, Jemal A. Cancer Statistics, 2017. CA: a cancer journal for clinicians 2017 1;67(1):7-30. [PubMed: 28055103]

2. Burnett AK, Russell NH, Hills RK, Kell J, Cavenagh J, Kjeldsen L, et al. A randomized comparison of daunorubicin $90 \mathrm{mg} / \mathrm{m} 2$ vs $60 \mathrm{mg} / \mathrm{m} 2$ in AML induction: results from the UK NCRI AML17 trial in 1206 patients. Blood 20156 18;125(25):3878-85. [PubMed: 25833957]

3. Fernandez HF, Sun Z, Yao X, Litzow MR, Luger SM, Paietta EM, et al. Anthracycline dose intensification in acute myeloid leukemia. The New England journal of medicine 20099 24;361(13):1249-59. [PubMed: 19776406]

4. Lee JH, Joo YD, Kim H, Bae SH, Kim MK, Zang DY, et al. A randomized trial comparing standard versus high-dose daunorubicin induction in patients with acute myeloid leukemia. Blood 201110 6;118(14):3832-41. [PubMed: 21828126]

5. Lowenberg B, Ossenkoppele GJ, van Putten W, Schouten HC, Graux C, Ferrant A, et al. High-dose daunorubicin in older patients with acute myeloid leukemia. The New England journal of medicine 20099 24;361(13):1235-48. [PubMed: 19776405]

6. Luskin MR, Lee JW, Fernandez HF, Abdel-Wahab O, Bennett JM, Ketterling RP, et al. Benefit of high-dose daunorubicin in AML induction extends across cytogenetic and molecular groups. Blood 20163 24;127(12):1551-8. [PubMed: 26755712]

7. Preisler H, Davis RB, Kirshner J, Dupre E, Richards F 3rd, Hoagland HC, et al. Comparison of three remission induction regimens and two postinduction strategies for the treatment of acute nonlymphocytic leukemia: a cancer and leukemia group B study. Blood 1987 5;69(5):1441-9. [PubMed: 3552076]

8. Berman E, Heller G, Santorsa J, McKenzie S, Gee T, Kempin S, et al. Results of a randomized trial comparing idarubicin and cytosine arabinoside with daunorubicin and cytosine arabinoside in adult 
patients with newly diagnosed acute myelogenous leukemia. Blood 19914 15;77(8):1666-74. [PubMed: 2015395]

9. Dohner H, Estey E, Grimwade D, Amadori S, Appelbaum FR, Buchner T, et al. Diagnosis and management of AML in adults: 2017 ELN recommendations from an international expert panel. Blood 20171 26;129(4):424-47. [PubMed: 27895058]

10. Miller KD, Siegel RL, Lin CC, Mariotto AB, Kramer JL, Rowland JH, et al. Cancer treatment and survivorship statistics, 2016. CA: a cancer journal for clinicians 2016 7;66(4):271-89. [PubMed: 27253694]

11. Damle NK, Frost P. Antibody-targeted chemotherapy with immunoconjugates of calicheamicin. Current opinion in pharmacology 2003 8;3(4):386-90. [PubMed: 12901947]

12. Ricart AD. Antibody-drug conjugates of calicheamicin derivative: gemtuzumab ozogamicin and inotuzumab ozogamicin. Clinical cancer research : an official journal of the American Association for Cancer Research 201110 15;17(20):6417-27. [PubMed: 22003069]

13. Linenberger ML. CD33-directed therapy with gemtuzumab ozogamicin in acute myeloid leukemia: progress in understanding cytotoxicity and potential mechanisms of drug resistance. Leukemia 2004 12/09/online;19(2):176-82.

*14. van Der Velden VH, te Marvelde JG, Hoogeveen PG, Bernstein ID, Houtsmuller AB, Berger MS, et al. Targeting of the CD33-calicheamicin immunoconjugate Mylotarg (CMA-676) in acute myeloid leukemia: in vivo and in vitro saturation and internalization by leukemic and normal myeloid cells. Blood 20015 15;97(10):3197-204. [PubMed: 11342449] - Reference detailing CD33 receptor recycling on the surface of AML blasts as providing the biological rationale for fractionating $\mathrm{GO}$ dosing for clinical usage

15. Mylotarg (gemtuzumab ozogamicin) [prescribing information] Philadelphia, PA: Wyeth Pharmaceuticals; 92017.

*16. FDA Oncologic Drugs Advisory Committee Briefing Document (BLA 761060) - Gemtuzumab Ozogamicin 20177 11, 2017 [cited 2018 March 3, 2018]; Available from: https://www.fda.gov/ downloads/AdvisoryCommittees/CommitteesMeetingMaterials/Drugs/ OncologicDrugsAdvisoryCommittee/UCM566015.pdf-- Reference providing updated analyses of pivotal GO therapeutic trials in AML, specifically the ALFA-0701 and meta-analysis data sets with longer follow-up.

**17. Larson RA, Sievers EL, Stadtmauer EA, Lowenberg B, Estey EH, Dombret H, et al. Final report of the efficacy and safety of gemtuzumab ozogamicin (Mylotarg) in patients with CD33-positive acute myeloid leukemia in first recurrence. Cancer 200510 1;104(7):1442-52. [PubMed: 16116598] - Description of the clinical trials with GO which led to its initial approval for relapsed/refractory AML patients in 2000

18. Bross PF, Beitz J, Chen G, Chen XH, Duffy E, Kieffer L, et al. Approval summary: gemtuzumab ozogamicin in relapsed acute myeloid leukemia. Clinical cancer research : an official journal of the American Association for Cancer Research 2001 6;7(6):1490-6. [PubMed: 11410481]

19. Arceci RJ, Sande J, Lange B, Shannon K, Franklin J, Hutchinson R, et al. Safety and efficacy of gemtuzumab ozogamicin in pediatric patients with advanced CD33+ acute myeloid leukemia. Blood 20058 15;106(4):1183-8. [PubMed: 15886328]

**20. Petersdorf SH, Kopecky KJ, Slovak M, Willman C, Nevill T, Brandwein J, et al. A phase 3 study of gemtuzumab ozogamicin during induction and postconsolidation therapy in younger patients with acute myeloid leukemia. Blood 20136 13;121(24):4854-60. [PubMed: 23591789] -- The phase 3 clinical trial of GO plus chemotherapy in younger AML patients which preliminarily reported increased mortality in the GO arm, leading to its premature withdrawal from the commerical market in 2010.

21. Richwine L Pfizer pulls Leukemia Drug from U.S. Market 2010 [cited 2017 October 15]; Available from: https://www.reuters.com/article/us-pfizer-mylotarg/pfizer-pulls-leukemia-drug-from-u-smarket-idUSTRE65K5QG20100621

**22. Taksin AL, Legrand O, Raffoux E, de Revel T, Thomas X, Contentin N, et al. High efficacy and safety profile of fractionated doses of Mylotarg as induction therapy in patients with relapsed acute myeloblastic leukemia: a prospective study of the alfa group. Leukemia 2007 1;21(1):6671. [PubMed: 17051246] - Results of the Mylo-France 1 trial which demonstrated improved 
clinical outcomes for relapsed/refractory AML patients treated with fractionated GO monotherapy

**23. Castaigne S, Pautas C, Terre C, Raffoux E, Bordessoule D, Bastie JN, et al. Effect of gemtuzumab ozogamicin on survival of adult patients with de-novo acute myeloid leukaemia (ALFA-0701): a randomised, open-label, phase 3 study. Lancet (London, England) 20124 21;379(9825):1508-16.- Results of the ALFA-0701 trial which demonstrated prolonged EFS when adding fractionated GO dosing to standard 7+3 chemotherapy for newly diagnosed AML patients. This was one of the clinical trials leading to re-approval of this agent in 2017.

24. Jen EY, Ko CW, Lee JE, Del Valle PL, Aydanian A, Jewell C, et al. FDA Approval: Gemtuzumab ozogamicin for the treatment of adults with newly-diagnosed CD33-positive acute myeloid leukemia. Clinical cancer research : an official journal of the American Association for Cancer Research 2018223.

25. Lambert J, Lambert J, Nibourel O, Pautas C, Hayette S, Cayuela JM, et al. MRD assessed by WT1 and NPM1 transcript levels identifies distinct outcomes in AML patients and is influenced by gemtuzumab ozogamicin. Oncotarget 20148 15;5(15):6280-8. [PubMed: 25026287]

26. Renneville A, Abdelali RB, Chevret S, Nibourel O, Cheok M, Pautas C, et al. Clinical impact of gene mutations and lesions detected by SNP-array karyotyping in acute myeloid leukemia patients in the context of gemtuzumab ozogamicin treatment: results of the ALFA-0701 trial. Oncotarget 20142 28;5(4):916-32. [PubMed: 24659740]

**27. Hills RK, Castaigne S, Appelbaum FR, Delaunay J, Petersdorf S, Othus M, et al. Addition of gemtuzumab ozogamicin to induction chemotherapy in adult patients with acute myeloid leukaemia: a meta-analysis of individual patient data from randomised controlled trials. The Lancet Oncology 2014 8;15(9):986-96. [PubMed: 25008258] - Meta-analysis of 3325 patients in five clinical trials of fractionated/lower dose GO demonstrating an improved EFS and OS when adding GO to standard chemotherapy for AMLpatients. This contributed to re-approval of the agent in 2017.

**28. Amadori S, Suciu S, Selleslag D, Aversa F, Gaidano G, Musso M, et al. Gemtuzumab Ozogamicin Versus Best Supportive Care in Older Patients With Newly Diagnosed Acute Myeloid Leukemia Unsuitable for Intensive Chemotherapy: Results of the Randomized Phase III EORTC-GIMEMA AML-19 Trial. Journal of clinical oncology : official journal of the American Society of Clinical Oncology 20163 20;34(9):972-9. [PubMed: 26811524] - Use of a divided dosing scheduled of GO $(6 \mathrm{mg} / \mathrm{m} 2$ followed by $3 \mathrm{mg} / \mathrm{m} 2)$ improved survival of older unfit AML patients as compared with best supportive care.

29. Lamba JK, Chauhan L, Shin M, Loken MR, Pollard JA, Wang YC, et al. CD33 Splicing Polymorphism Determines Gemtuzumab Ozogamicin Response in De Novo Acute Myeloid Leukemia: Report From Randomized Phase III Children's Oncology Group Trial AAML0531. Journal of clinical oncology : official journal of the American Society of Clinical Oncology 20178 10;35(23):2674-82. [PubMed: 28644774]

30. Gale RE, Popa T, Wright M, Khan N, Freeman SD, Burnett AK, et al. No evidence that CD33 splicing SNP impacts the response to GO in younger adults with AML treated on UK MRC/NCRI trials. Blood 20181 25;131(4):468-71. [PubMed: 29229596]

31. Pollard JA, Loken M, Gerbing RB, Raimondi SC, Hirsch BA, Aplenc R, et al. CD33 Expression and Its Association With Gemtuzumab Ozogamicin Response: Results From the Randomized Phase III Children's Oncology Group Trial AAML0531. Journal of clinical oncology : official journal of the American Society of Clinical Oncology 20163 1;34(7):747-55. [PubMed: 26786921]

32. Olombel G, Guerin E, Guy J, Perrot JY, Dumezy F, de Labarthe A, et al. The level of blast CD33 expression positively impacts the effect of gemtuzumab ozogamicin in patients with acute myeloid leukemia. Blood 20164 28;127(17):2157-60. [PubMed: 26929274]

33. Goemans BF, Zwaan CM, Vijverberg SJ, Loonen AH, Creutzig U, Hahlen K, et al. Large interindividual differences in cellular sensitivity to calicheamicin may influence gemtuzumab ozogamicin response in acute myeloid leukemia. Leukemia 2008 12;22(12):2284-5. [PubMed: 18563175] 
34. Bertoli S, Berard E, Huguet F, Huynh A, Tavitian S, Vergez F, et al. Time from diagnosis to intensive chemotherapy initiation does not adversely impact the outcome of patients with acute myeloid leukemia. Blood 20134 4;121(14):2618-26. [PubMed: 23365464]

35. Fenaux P, Mufti GJ, Hellstrom-Lindberg E, Santini V, Gattermann N, Germing U, et al. Azacitidine prolongs overall survival compared with conventional care regimens in elderly patients with low bone marrow blast count acute myeloid leukemia. Journal of clinical oncology : official journal of the American Society of Clinical Oncology 20102 1;28(4):562-9. [PubMed: 20026804]

36. Kantarjian HM, Thomas XG, Dmoszynska A, Wierzbowska A, Mazur G, Mayer J, et al. Multicenter, randomized, open-label, phase III trial of decitabine versus patient choice, with physician advice, of either supportive care or low-dose cytarabine for the treatment of older patients with newly diagnosed acute myeloid leukemia. Journal of clinical oncology : official journal of the American Society of Clinical Oncology 20127 20;30(21):2670-7. [PubMed: 22689805]

37. Blum W, Garzon R, Klisovic RB, Schwind S, Walker A, Geyer S, et al. Clinical response and miR-29b predictive significance in older AML patients treated with a 10-day schedule of decitabine. Proceedings of the National Academy of Sciences of the United States of America 20104 20;107(16):7473-8. [PubMed: 20368434]

38. Welch JS, Petti AA, Miller CA, Fronick CC, O'Laughlin M, Fulton RS, et al. TP53 and Decitabine in Acute Myeloid Leukemia and Myelodysplastic Syndromes. The New England journal of medicine 201611 24;375(21):2023-36. [PubMed: 27959731]

39. Daver N, Kantarjian H, Ravandi F, Estey E, Wang X, Garcia-Manero G, et al. A phase II study of decitabine and gemtuzumab ozogamicin in newly diagnosed and relapsed acute myeloid leukemia and high-risk myelodysplastic syndrome. Leukemia 2016 2;30(2):268-73. [PubMed: 26365212]

40. Nand S, Othus M, Godwin JE, Willman CL, Norwood TH, Howard DS, et al. A phase 2 trial of azacitidine and gemtuzumab ozogamicin therapy in older patients with acute myeloid leukemia. Blood 201311 14;122(20):3432-9. [PubMed: 24092933]

41. Candoni A, Papayannidis C, Martinelli G, Simeone E, Gottardi M, Iacobucci I, et al. Flai (fludarabine, cytarabine, idarubicin) plus low-dose gemtuzumab ozogamicin as induction therapy in cd33-positive aml: Final results and long term outcome of a phase ii multicenter clinical trial. American journal of hematology 201822.

42. Wang ES, Zeidan A, Tan W, Wilding GE, Ford LA, Wallace PK, et al. Cytoreduction with gemtuzumab ozogamicin and cytarabine prior to allogeneic stem cell transplant for relapsed/ refractory acute myeloid leukemia. Leukemia \& lymphoma 2012 10;53(10):2085-8. [PubMed: 21740302]

43. Wattad M, Weber D, Dohner K, Krauter J, Gaidzik VI, Paschka P, et al. Impact of salvage regimens on response and overall survival in acute myeloid leukemia with induction failure. Leukemia 2017 6;31(6):1306-13. [PubMed: 28138160]

44. Marcucci G, Geyer S, Zhao W, Caroll AJ, Bucci D, Uy GL, et al. Adding KIT Inhibitor Dasatinib (DAS) to Chemotherapy Overcomes the Negative Impact of KIT Mutation/over-Expression in Core Binding Factor (CBF) Acute Myeloid Leukemia (AML): Results from CALGB 10801 (Alliance). Blood 2014;124(21):8-. 
9.

\section{Key Issues}

- $\mathrm{GO}$ is a monoclonal antibody-drug conjugate (ADC) consisting of an monoclonal antibody, (gemtuzumab) targeting human CD33 attached via a linker to the cytotoxic derivative of calicheamicin (ozogamicin) with DNAdamaging effects

- $\quad$ GO received initial FDA approval in 2000 based on a $9 \mathrm{mg} / \mathrm{m}^{2}$ dosing regimen in RR- AML; however it was subsequently withdrawn from the US marketplace due to preliminary results of a phase 3, randomized, controlled trial (SWOG-S0106) which demonstrated increased early deaths and lack of clinical benefit in the GO + chemotherapy arm.

- Administration of GO at lower doses $\left(3-6 \mathrm{mg} / \mathrm{m}^{2}\right)$ on fractionated dosing schedules (days 1, 4, 7 or days 1 and 8) has resulted in improved clinical efficacy with decreased hepatotoxicity and veno-occlusive disease

- For RR-AML, single-agent GO resulted in a 33\% overall response rate and prolonged relapse-free survival (median 11.0 months) in responding patients. Dosing of GO is $3 \mathrm{mg} / \mathrm{m}^{2}$ on days 1,4 and 7 .

- For ND-AML patients unfit for intensive chemotherapy, single agent GO improved overall survival ( 4.9 vs. 3.6 months) over best supportive care. Dosing of GO is $6 \mathrm{mg} / \mathrm{m}^{2}$ on day 1 and $3 \mathrm{mg} / \mathrm{m}^{2}$ on day 8 during induction. Responding patients can receive GO dosed at $2 \mathrm{mg} / \mathrm{m}^{2}$ on day 1 every 4 weeks for up to 8 cycles of maintenance.

- $\quad$ For ND-AML fit for intensive chemotherapy, fractionated doses GO added to cytarabine and daunorubicin chemotherapy improved EFS (17.3 vs. 9.5 months). Dosing of GO is $3 \mathrm{mg} / \mathrm{m}^{2}$ on days 1,4 , and 7 of induction chemotherapy and $3 \mathrm{mg} / \mathrm{m}^{2}$ on day 1 of up to two consolidation chemotherapy courses.

- Optimal use of GO in the upfront AML setting requires rapid cytogenetic and molecular information in order to select for patients with favorable and intermediate (not adverse) risk cytogenetics most likely to benefit from addition of GO.

- $\quad \mathrm{GO}$ results in increased rates of hepatotoxicity (including a $<5 \%$ incidence of severe and fatal VOD/SOS), infusion related reactions (including anaphylaxis), and myelosuppression with prolonged cytopenias. It is important to monitor liver function and complete cell counts frequently during therapy, particularly in patients with baseline abnormal liver tests and with prior allogeneic stem cell transplantation.

- $\quad$ Novel biomarkers including CD33 expression on AML blasts and single nucleotide polymorphisms (SNPs) for the CD33 receptor hold promise in 
delineating which AML patients are most likely to most benefit from GO therapy. 


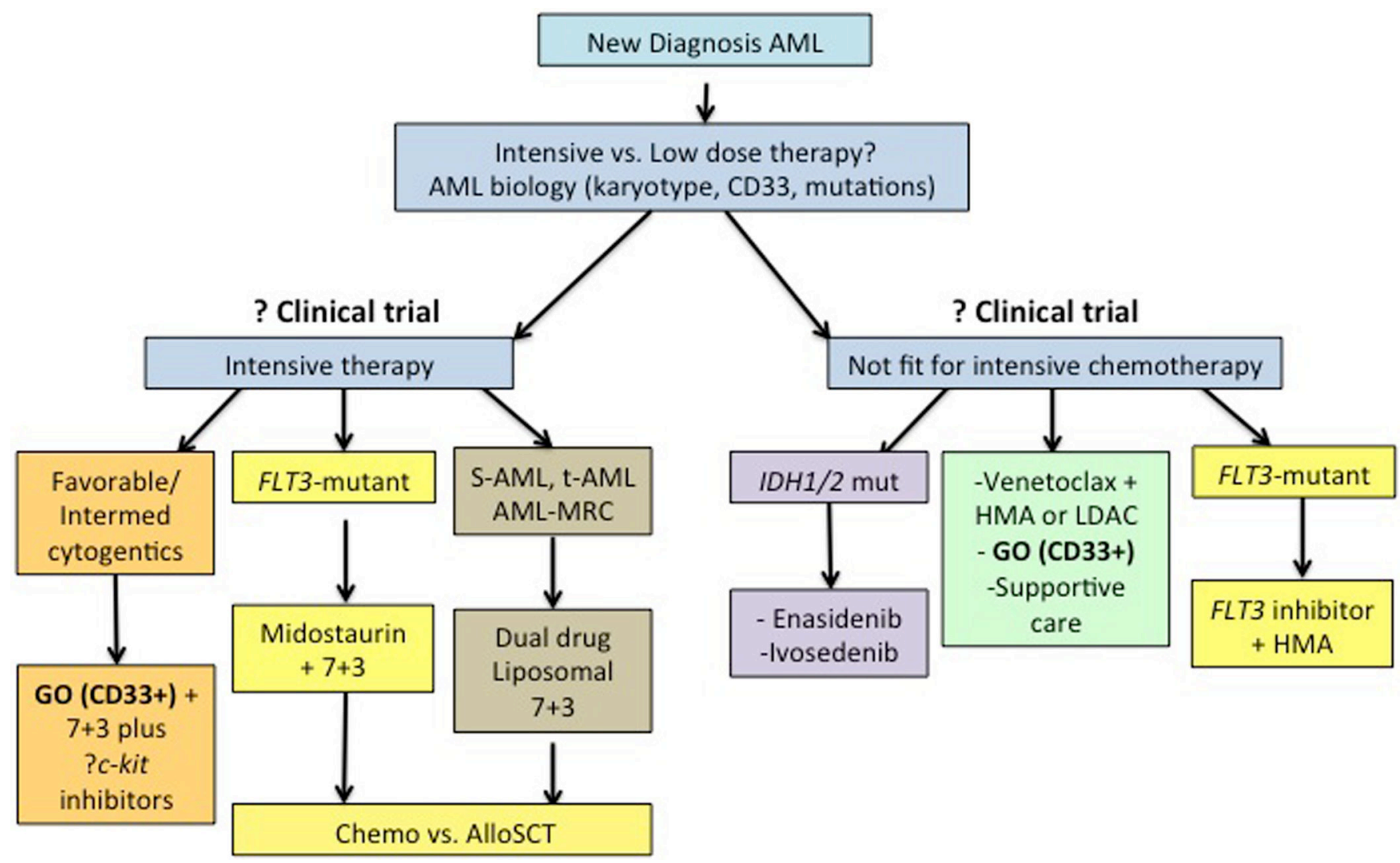

Figure 1.

Proposed treatment landscape for newly diagnosed AML 


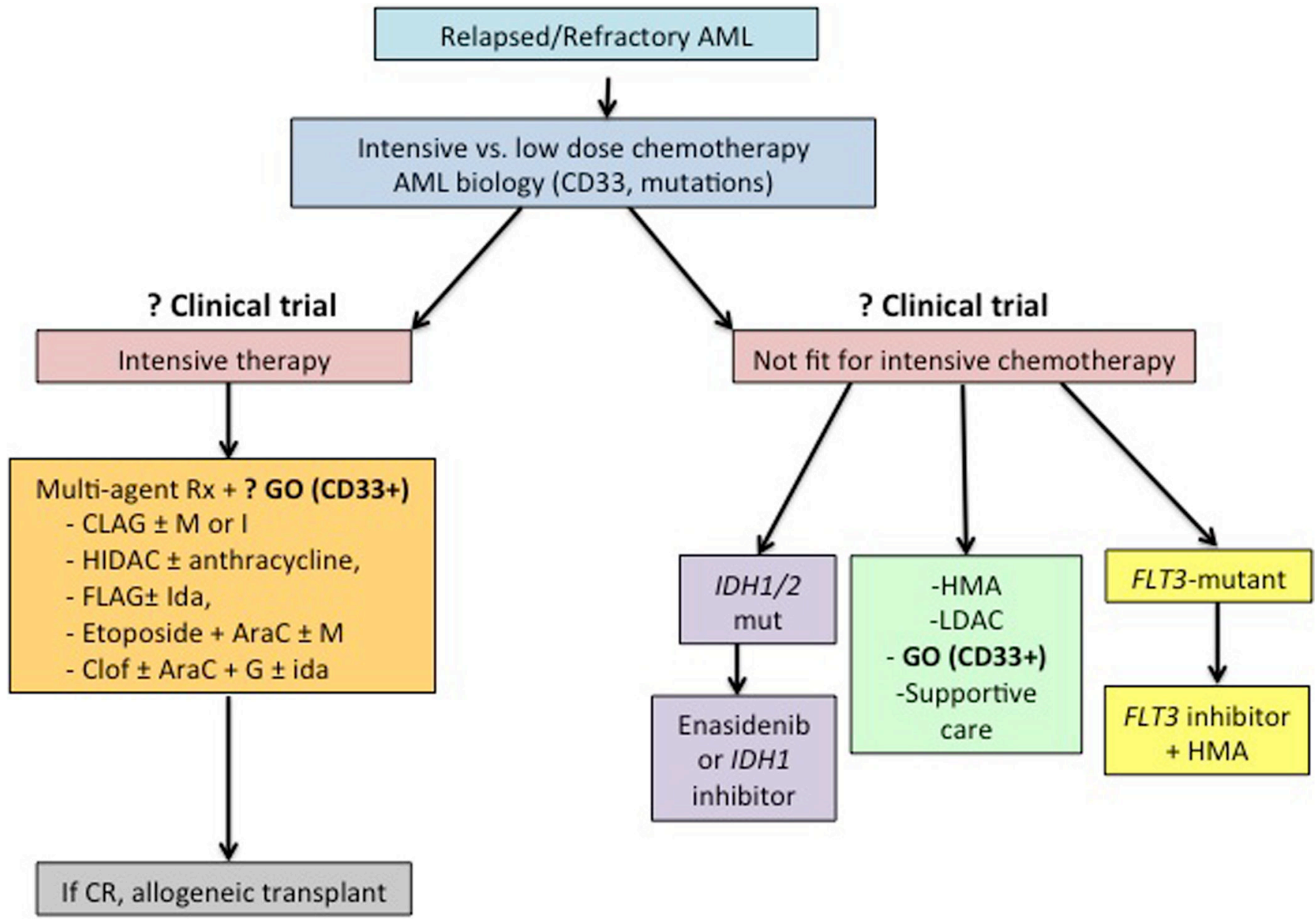

Figure 2.

Proposed treatment landscape for relapsed/refractory AML 


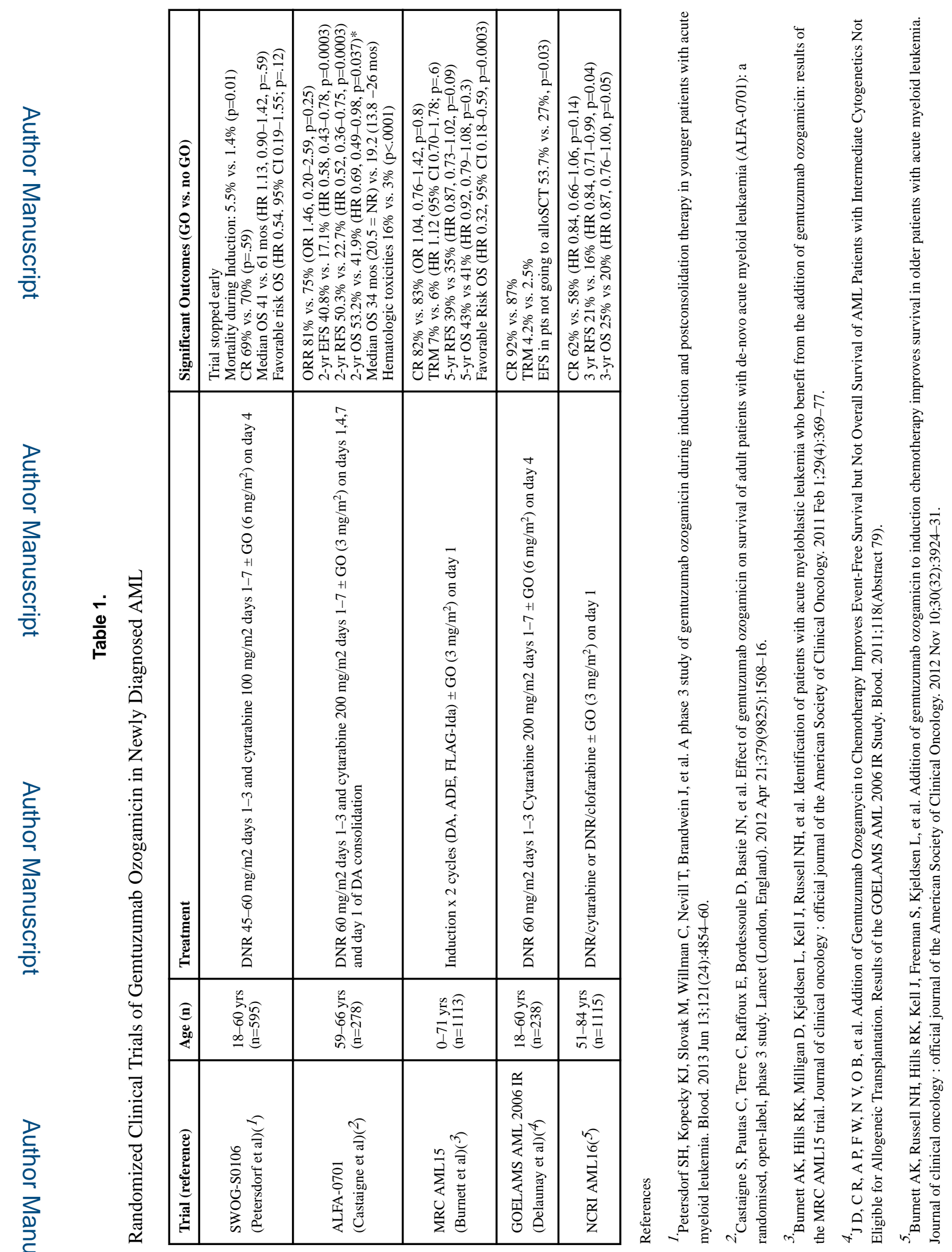

Expert Rev Clin Pharmacol. Author manuscript; available in PMC 2019 July 29. 


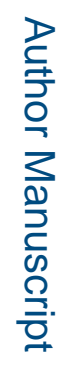

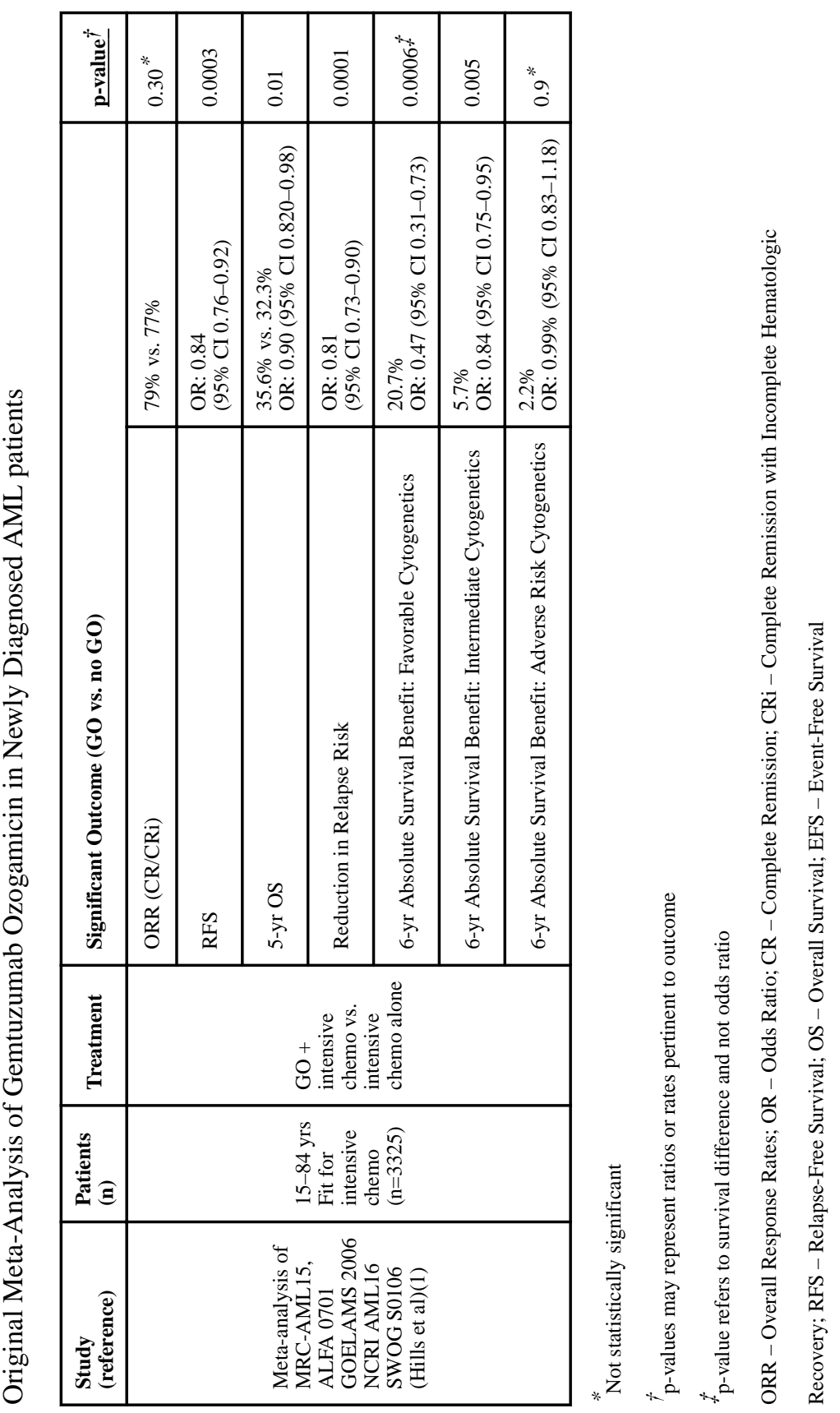

Expert Rev Clin Pharmacol. Author manuscript; available in PMC 2019 July 29. 


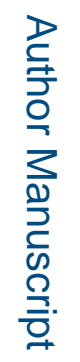

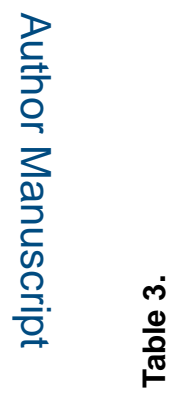

롤

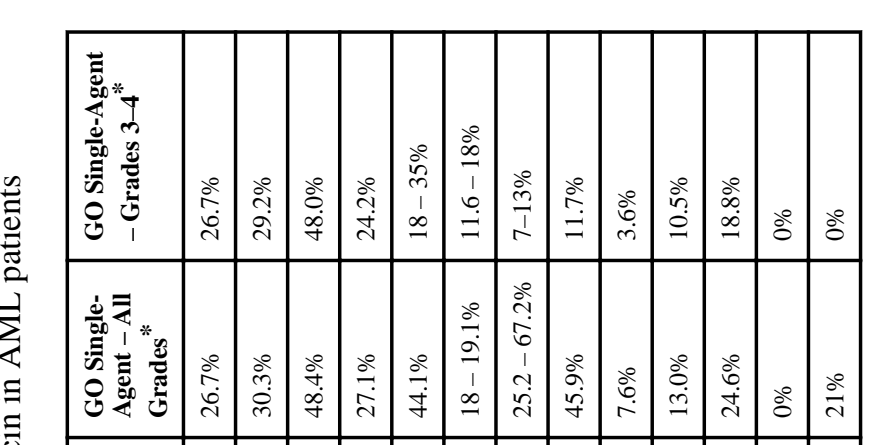

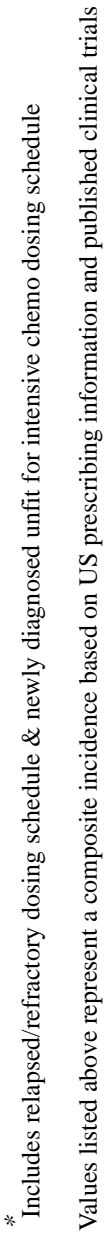

Expert Rev Clin Pharmacol. Author manuscript; available in PMC 2019 July 29. 


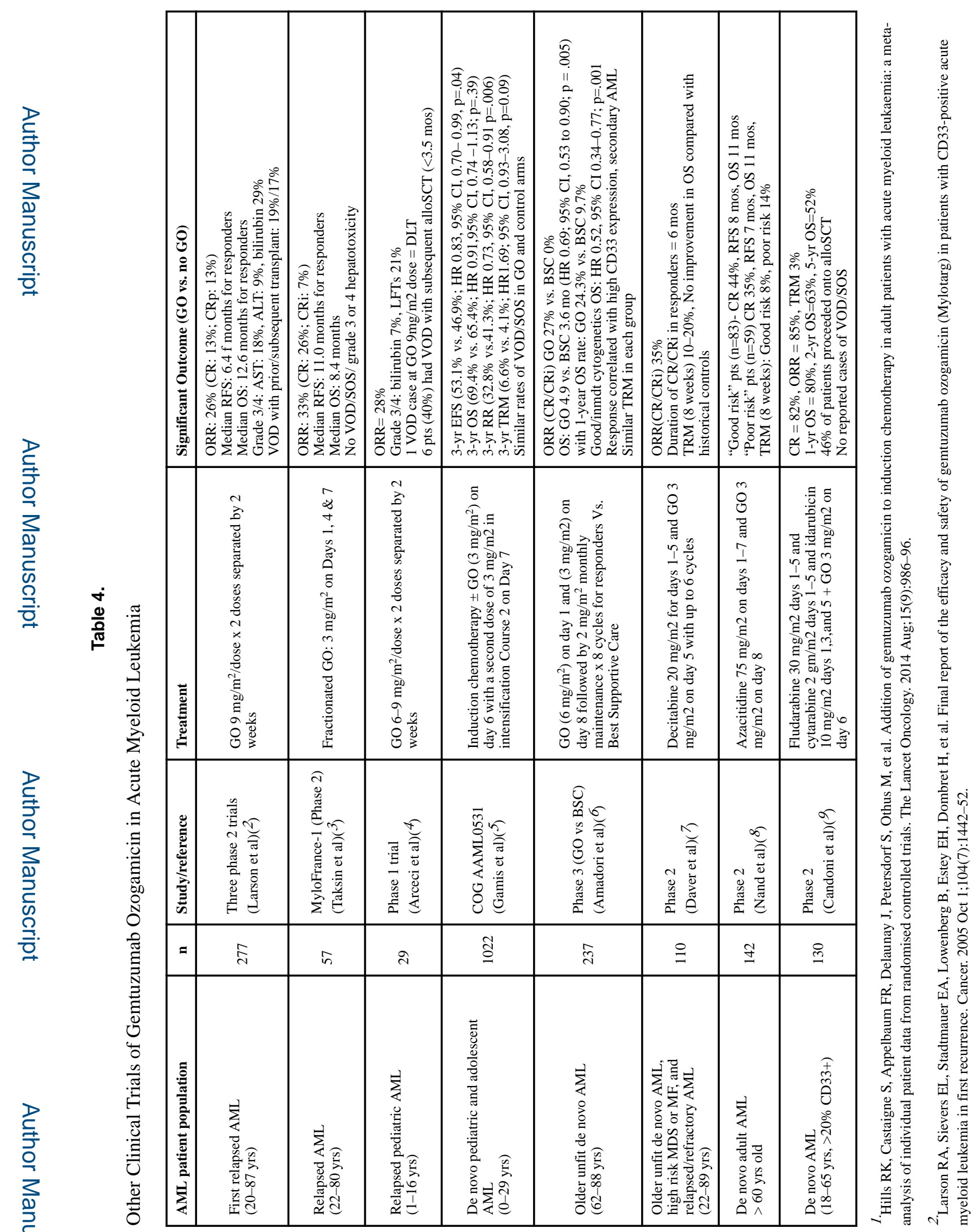

Expert Rev Clin Pharmacol. Author manuscript; available in PMC 2019 July 29. 


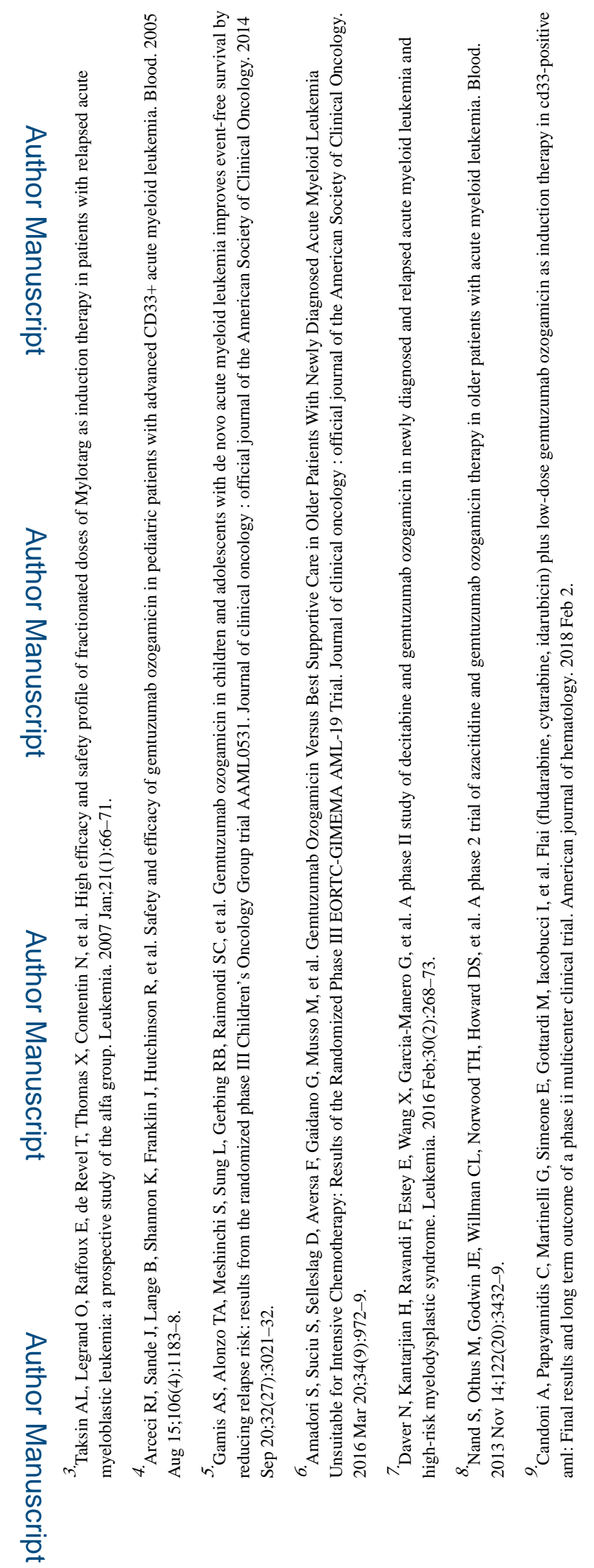

Expert Rev Clin Pharmacol. Author manuscript; available in PMC 2019 July 29. 